\title{
Role of the oral mucosa in the invasive infection by Candida parapsilsosis sensu stricto, under the condition of dysbiosis
}

\author{
Rodríguez $\mathrm{ML}^{1,2 *}$, Rosa $\mathrm{AC}^{3}$, Nastri $\mathrm{L}^{3}$ and Jewtuchowicz $\mathrm{VM}^{1,4}$ \\ ${ }^{1}$ University of Buenos Aires, School of Medicine, Institute of Research in Microbiology, Parasitology and Inmunology (IMPaM), Mycology Center. Buenos Aires- \\ Argentina \\ ${ }^{2}$ University of Cuenca, School of Dentistry, Department of Semiology and Diagnostic Clinic. Cuenca-Ecuador \\ ${ }^{3}$ University of Buenos Aires, School of Dentistry, Department of Microbiology and Parasitology. Buenos Aires-Argentina \\ ${ }^{4}$ HIGA Gandulfo Hospital, Laboratory Service. Buenos Aires Province-Argentina
}

\begin{abstract}
Over the past 10 years, there have been increasingly frequent reports of colonization of niches in the oral cavity by non-Candida albicans Candida (NCAC) species. Among these species, Candida parapsilosis sensu stricto plays a major part both locally and systemically. Evidence shows that it may be the second most frequent yeast isolate, after Candida albicans, as commensal in the oral cavity and as pathogen in blood cultures of patients diagnosed with fungemia. Recent reports suggest that both its distribution and its virulence may be affected by environmental conditions and may increase in situations of oral dysbiosis. Based on this background, we hypothesize that oral mucosa in pathological condition may be a source of candidemia by Candida parapsilosis sensu stricto, when promoted by the conditions in the host. We reviewed the scientific databases in search of evidence which may support such hypothesis.
\end{abstract}

\section{Introduction}

Candida parapsilosis sensu stricto belongs to the Candida parapsilosis complex along with two other species, Candida orthopsilosis and Candida metapsilosis. Although the phenotypes of these three species are very similar [1], the phylogenetic distance between them led to their being recorded as different species in 2005 [2]. The yeast Candida parapsilosis sensu stricto has been of medical interest over the past 10 years because it is the species most frequently isolated, after Candida albicans, in blood cultures of subjects diagnosed with candidemia, both adults [3] and preterm neonates [4], particularly in Europe [5], Asia [6] and Latin America [7].

Invasive candidiasis is the most common fungal infection in hospitalized patients. Indeed, Candida spp. is the fourth cause of nosocomial bloodstream infections [8]. Despite progress in anti-fungal therapy, candidemia continues to contribute to high mortality rates, lengthy hospitalizations and high healthcare costs $[9,10]$.

A change in the etiology of candidemia has been reported within the past decade. Whereas Candida albicans is still considered to be the most frequent cause of fungemia, higher rates of Candida tropicalis, Candida parapsilosis, Candida glabrata and Candida krusei in candidemia have been reported around the world [11-14].

Candida parapsilosis sensu stricto has also acquired clinical importance in the field of stomatology. Some studies based on cultures and others based on DNA have reported this species as the second most frequent yeast isolate from the oral cavity, after Candida albicans. The first information on this epidemiological trend was reported in 1996 by Kleinneger et al. who conducted a study on 172 white Americans in different age groups with good health status [15]. The same trend was reported in 2010 by Ghannoun et al. in a study based on DNA analysis by multi-tag pyrosequencing [16]. In 2011, Yang et al. reported a similar situation in a sample of 323 healthy Thai volunteers [17] Peters et al. recently published the results of a study based on DNA analysis by a massive sequencing technique to establish the fungal component in oral microbiota in subjects with periodontal disease vs. subjects with good oral health. Surprisingly, the paper reported lower colonization frequency for Candida albicans, with Candida quercitrusa and Candida parapsilosis being the two most frequently recovered fungi for both clinical conditions studied [18]. The information in the above paragraph shows that there has been a change in the composition of the oral mycobiome in recent years, with an increase in non-Candida albicans Candida (NCAC) species. In this group, Candida parapsilosis has emerged in the past decade as a major nosocomial pathogen [19]. According to current evidence, it is considered to be the most pathogenic and most prevalent species in the Candida parapsilsosis complex in

${ }^{*}$ Correspondence to: Rodríguez ML, University of Buenos Aires, School of Medicine, Institute of Research in Microbiology, Parasitology and Inmunology (IMPaM), Mycology Center. Buenos Aires-Argentina; University of Cuenca, School of Dentistry, Department of Semiology and Diagnostic Clinic. CuencaEcuador, E-mail: malourdes84@hotmail.com

Key words: oral candida, candida parapsilosis complex, candida parapsilosis sensu stricto, gingival-periodontal disease, biofilm, invasive candidiasis, bacteremia, symbiont, pathobiont

Received: February 12, 2019; Accepted: February 28, 2019; Published: March 04,2019 
the different human ecological niches [20-25]. Recent experimental evidence suggests that the recovery rate of Candida parapsilosis sensu stricto is higher in oral cavities under inflammatory conditions [26]. The species has also been shown to have a more pathogenic phenotype in vitro when it comes from an inflammatory oral environment [27].

This paper therefore provides a review of the literature with the aim of presenting evidence currently supporting the hypothesis that oral mucosa under pathological conditions is a potential source of candidemia by Candida parapsilosis sensu stricto.

\section{Materials and methods}

A PubMed and Google scholar scientific database search was done using the following key words: Oral Candida; Candida parapsilosis complex; Candida parapsilosis sensu stricto; Gingival-periodontal disease; Biofilm; Invasive candidiasis; Bacteremia; Symbiont and Pathobiont. Articles published over the past 15 years with different methodological designs were selected. Clinical and basic research papers dealing with the following topics were selected: invasive candidiasis; candidemia epidemiology; Candida parapsilosis complex: Candida parapsilosis sensu stricto and its behavior in the oral cavity; fungal component of human oral microbiota; biofilm of oral isolates of Candida parapsilosis sensu stricto; Oral colonization by Candida and subsequent events of candidemia, and C. parapsilosis complex in oral colonization and disease in inmunocompetent and immunocompromised patients.

\section{Results}

The following evidence supporting the hypothesis of the current paper was found in the literature:

1. Fanello, et al., (Journal of Medical Microbiology) [28] conducted a prospective longitudinal clinical investigation on 256 patients in the Internal Medicine area of a geriatric hospital in Paris (156 women and 100 men, average age $83 \pm 8$ years). The subjects were studied to determine colonization or infection by yeasts. A significant association was found between oral Candida genus yeast carriage density and the onset of a nosocomial fungal infection, either invasive or non-invasive. The study found high prevalence of oral colonization by yeasts $(67 \%)$ on day 0 , while $51 \%$ of the subjects had high colonization ( $>50$ c.f.u). Three risk factors were found for oral colonization with Candida genus yeasts: a) poor oral hygiene ( $\mathrm{p}=0.002) ; \mathrm{b})$ use of peripheral catheter $(\mathrm{p}=0.014)$; and $\mathrm{c}$ ) use of dental prosthesis $(p=0.03)$. Four risk factors were found for nosocomial fungal infection: a) high colony count in oral cavity $(>50$ c.f.u) ( $\mathrm{p}=0.0001)$; b) malnutrition ( $\mathrm{p}=0.006)$; c) endocrine disorders $(\mathrm{p}=0.027)$; and $\mathrm{d})$ prolonged hospitalization $(\mathrm{p}=0.008)$. Candida albicans accounted for $66 \%$ of isolates, followed by Candida glabrata and Candida tropicalis.

The study concluded that there is a link among colonization, carriage density and fungal infection. It suggests that oral samples should be collected on day 0 of hospitalization and antifungal treatment administered in cases of high density of colonization.

It would be important to conduct studies with similar methodological design in other geographical regions in order to establish worldwide consensuses on hospitalization protocols in critical and non-critical areas.

2. Rubio, et al., (Revista Iberoamericana de Micología) [29] demonstrated by means of a cross-sectional prospective clinical study that under conditions of gingivo-periodontal disease, the oral cavity becomes a favorable niche for colonization, proliferation and expression of a pathogenic phenotype of different Candida species, promoting invasion of the connective tissue. The study included 55 patients of both sexes, 21 to 55 years old, with gingivo-periodontal diseases, immunocompetent, non-smokers, and without prior antimicrobial treatment. Samples were taken of soft tissues, supragingival and subgingival plaque, and biopsies of periodontal pockets. Microscopic studies, cultures, assimilation profiles and DNA amplification were performed. The study concluded that the different Candida species may form part of periodontal microbial plaque and play a major role in its adherence to tissues, enabling indepth invasion. They may also infect subgingival niches in patients with gingivitis, including healthy sites, acting as commensals or opportunists.

3. Batista, et al., (Journal of Medical Mycology) [30] conducted a prospective longitudinal clinical study, for 6 months, on 125 neonates at high risk for nosocomial infection, to be studied for oral colonization and fungal septicemia. From this total, 19 neonates had yeasts on oral mucosa, and 12 developed fungemia. Concordance was found between yeasts colonizing the oral mucosa and those in the blood in $6(50 \%)$ of the 12 neonates who developed fungemia. The yeasts isolated in the 6 cases with concordance of Candida species between oral mucosa and blood were Candida albicans in 5 cases (83.4\%), and Candida parapsilosis in only one case, accounting for $16.6 \%$. Pulsed-field gel electrophoresis showed that all 6 yeast strains involved in oral colonization and blood had the same karyotype. Over the 6 months of follow-up, $66.6 \%$ of the patients with concordance of yeast species and genotypes between the oral mucosa and the blood progressed to death. The study suggests the relevance of monitoring the oral microbiota and considering it as a possible source of infection.

The results of this study are supported by others which have shown that colonization is the main factor leading to development of infection, while the characteristics of the patient and the yeasts determine whether or not the infection evolves to sepsis $[28,31]$.

This was a high impact study, not only due to its methodological design, but also because of the results, confirming findings in other studies which have reported both Candida albicans and Candida parapsilosis as the two Candida species frequently involved in systemic diseases caused by fungi in neonates [32,33].

We believe that the study should be replicated on a larger sample, with participation of several hospitals and/or several countries or regions.

4. Rodríguez L, et al., (Journal of Dental Science and Therapy) [26] demonstrated by means of a basic, descriptive, cross-sectional, retrospective study that in the Candida parapsilosis complex, the species C. parapsilosis sensu stricto may behave as a usual colonizer in oral mucosa sites, predominating in inflammatory conditions and in presence of appliances, with statistically significant association. The study also suggested that the oral cavity may be a reservoir for strains of Candida parapsilosis sensu stricto with resistant phenotype and/or reduced susceptibility to the group of azoles and echinocandins. The study used 31 yeasts recognized according to phenotype as C. parapsilosis and recovered by endpoint PCR molecular characterization with specific primers. It also evaluated in vitro sensitivity against azoles, echinocandins, amphotericin B and flucytosine by automated Vitek2 system in line with CLSI guideline M27-A3. One hundred percent of the strains were confirmed by molecular biology as Candida parapsilosis sensu stricto, of which 
77.4\% were recovered in inflammatory conditions, while only $5.3 \%$ of the strains showed resistant phenotype to the group of azoles and echinocandins.

The results reported in this study regarding distribution of species in the C. parapsilosis complex in oral niches are compatible with those reported by other authors such as Urzúa, et al., [34] Ghannoum M, et al., [16]; Yang, et al., [17]; Laurenco A, et al., [35]; and Peters, et al., [18].

The weaknesses in this study are mainly the low sample size and the fact that it is retrospective, since this type of study usually causes information bias. However, the results are very interesting and worth confirming through a prospective study and using a larger number of isolates.

5. Rodríguez L, et al., (Journal of Dental Science and Therapy) [27] conducted a study to analyze in vitro virulence of 28 C. parapsilosis sensu stricto oral isolates, of which 21 came from pathological niches (periodontal disease) and 7 from subjects with good oral health. Virulence was measured by in vitro biofilm-forming capacity using the crystal violet technique on 96-well polystyrene plates; under two different nutritional conditions: YPD broth and RPMI 1640 medium. Each strain was run in 8 replicates and two independent experiments. The study showed a statistically significant difference in biofilm-forming capacity between strains obtained from oral cavities in dysbiosis and strains obtained from healthy oral cavities for both nutritional conditions tested. Interestingly, over $70 \%$ of the isolates showed high biofilm-forming capacity in both culture media. These findings suggest that the oral cavity may be colonized by Candida parapsilosis strains which may behave either as symbionts or as pathobionts according to environmental circumstances.

The results of this study are unprecedented, since there is no other published study with similar design or aimed at specifically studying the behavior of this Candida strain in the oral cavity. We consider that it is essential to replicate this methodological design in other geographic regions and using a greater number of clinical isolates. It would be important to complement the crystal violet test results with a technique to measure biofilm by metabolic activity, such as XTT, and to evaluate the virulence of this Candida species at transcriptional level.

6. Gingival-Periodontal disease is a set of inflammatory conditions that affect the gingiva and the apparatus for the insertion of the tooth (Gingivitis and periodontitis), of polymicrobial nature, and of multifactorial etiopathogenesis; can lead to the loss of teeth and contribute to systemic inflammation, affecting the quality of life of patients who suffer from it $[36,37]$.

Periodontitis is a chronic non-communicable disease (NCD) that shares social determinants and risk factors with the major NCDs that cause around two-thirds of deaths such as heart disease, diabetes, cancer and chronic respiratory disease [38-42]. Besides the shared risk factors with major NCDs, accumulation of dental biofilms consequent to inadequate self-performed oral hygiene procedures, such as tooth brushing and use of inter-dental cleaning aids, accounts for the initiation and progression of periodontal disease in the population. These biofilms, if not well controlled, interact with the unique susceptibility profile of each individual and may become dysbiotic, thereby initiating and sustaining the disease process which is characterized by the inflammatory destruction of the tooth-supporting apparatus and alveolar bone [43-46]. The key presentations of periodontitis in the early stages are gingival bleeding, recession of the gingival margin and halitosis. Measurable changes in oral health-related quality of life are present $[47,48]$.

The study global burden disease (GBD) 2010 reported that, in 2010, severe periodontitis was the sixth most prevalent condition and that it affected $10.8 \%$ (95\% UI: 10.1-11.6) or 743 million people aged 15-99 worldwide. The authors reported that the age-standardized prevalence of severe periodontitis in the global population had remained static over the previous two decades at $11.2 \%$ (95\% UI: 10.4-11.9 in 1990 and $10.5 \%$ to $12.0 \%$ in 2010). Similarly, the age-standardized incidence of severe periodontitis had not changed significantly between 1990 and 2010, being 701 cases per 100,000 person-years in 2010 (95\% UI: 599823 ) and 696 cases per 100,000 person-years in 1990 (95\% UI: 604808). These age-standardized prevalence's and incidences were similar for males and females. The prevalence of severe periodontitis increased with age, with a steep increase between the third and fourth decades of life, reaching peak prevalence at the age of 40 and remaining stable thereafter. There was a peak in incidence at age 38. Again, globally, these patterns did not change between 1990 and 2010. The authors highlighted the variations by country and world region, with the lowest prevalence of severe periodontitis being 4.5\% in Oceania in 2010 (95\% UI: 2.4-7.2) and the highest prevalence of severe periodontitis being 20.4\% in Southern Latin America in 2010 (95\% UI: 12.3-31.4). These regions also had the lowest and highest incidence of periodontitis in 2010 of 253 cases per 100,000 person-years (95\% UI: 160-393) and 1427 cases per 100,000 person-years (95\% UI: 922-2254). Between 1990 and 2010, there was no appreciable change in prevalence or incidence of periodontitis in any of the world's regions [49]

Considerable evidence also points to the fact that the effects of periodontitis go beyond the oral cavity and that the body is affected by the haematogenous dissemination of both bacteria and bacterial products originating in the oral biofilms and inflammatory mediators originating in the inflamed periodontium. Through these mechanisms, periodontitis interacts with various systemic diseases, notably diabetes, atherosclerosis, rheumatoid arthritis and pulmonary infections. Loss of masticatory function consequent to periodontal attachment loss and tooth loss due to severe periodontitis impacts on nutrition, as subjects with masticatory dysfunction change their dietary habits usually incorporating more starch and fats and less fresh fruit and vegetables in their diet. The systemic inflammatory burden and poor nutrition consequent to severe periodontitis may contribute to the pathogenesis of chronic NCDs [50].

7. The American Heart Association published a meta-analysis in 2007 (Journal of the American Heart Association) [51], establishing that transitory bacteremia events are common with manipulation of the tooth-gum complex, with frequencies in different reports ranging from 10 to $100 \%$ for tooth extraction; 36 to $88 \%$ for periodontal surgery; 8 to $80 \%$ for scaling and root planing; $40 \%$ for dental prophylaxis; and $20 \%$ for endodontic procedures. However, this meta-analysis also shows that transitory bacteremia occurs with routine activities such as tooth brushing, use of dental floss (20 to $68 \%$ ) and mastication (7 to $51 \%$ ); and considering that average number of visits to the dentist per person in USA is less than twice a year, the frequency of bacteremia from routine activities is much more significant.

According to evidence gathered by this analysis, it is estimated that tooth brushing twice a day for 1 year poses a risk 154,000 times higher of exposure to bacteremia than the risk arising from a simple tooth extraction, and that cumulative exposure over 1 year to 
bacteremia from routine activities may be as much as 5.6 million times higher than during a tooth extraction, which is considered the type of procedure most likely to cause bacteremia [51].

The issues raised in this AHA meta-analysis are very important from a clinical standpoint, considering that the oral cavity in pathological condition can to be a usual reservoir of Candida parapsilosis sensu stricto strains with pathogenic potential, which means that every time a subject with periodontal disease brushes or cleans his/her teeth, and every time he/she chews, he/she is causing a bacteremia event, potentially releasing into the blood Candida strains with more or less potential for virulence, and that under certain host conditions, the clinical outcome could translate into invasive candidiasis. So far there are no association studies (cohorts and control cases) that allow us to consider periodontal disease as a risk factor for invasive candidiasis. The only data available so far is the work of Debelian, et al. who, through a prospective, transversal and descriptive study of basic research, demonstrated that demonstrated that endodontic treatment can be the cause of anaerobic bacteremia and fungemia. The aim of study was to use phenotypic and genetic methods to trace microorganisms released into the bloodstream during and after endodontic treatment back to their presumed source-the root canal. Microbiological samples were taken from the root canals of 26 patients with asymptomatic apical periodontitis of single-rooted teeth. All root canals contained anaerobic bacteria. The frequency of bacteremia varied from $31 \%$ to $54 \%$. The microorganisms from the root canal and blood presented identical phenotypic and genetic characteristics within the patients examined. In this study, the only fungus that could be detected both in blood and in the root canal was Saccharomyces cereviciae [52].

\section{Discussion}

The importance of Candida species as a cause of blood stream infections (BSI) has been highlighted in many studies in the past few years. In a 7-year long study analyzing nosocomial BSI in hospitals in the United States (Surveillance and Control of Pathogens of Epidemiological Importance [SCOPE]), Candida species was found to be the fourth most common cause of BSI in a hospital setup; and an increase in the incidence rate of candidemia has been reported from other parts of the world as well [53].

In the past two decades, a variety of factors like the Acquired Immuno-Deficiency Syndrome (AIDS) epidemic, increased number of patients receiving immunosuppressive therapy for transplantation, the increasing use of antimicrobials in the hospital setups and even in the community have played a key role in altering the epidemiology of invasive fungal infections in general and of candidemia in particular. Many studies have established independent risk factors for candidemia on the basis of multivariate analyses. The important independent risk factors include use of broad-spectrum antimicrobials, cancer chemotherapy, mucosal colonization by Candida species, indwelling vascular catheters like central venous catheters (CVCs) and others [54].

Respect to Candida colonization and candidemia, the source of BSI with Candida species has been a subject of considerable debate in the last couple of decades. Two major sources of infection have been proposed-the gastrointestinal tract (endogenous infection) and the skin (exogenous infection). In the past few years, however, there has been ample evidence pointing towards an endogenous, gastrointestinal origin for candidemia, and for some species of Candida like C. parapsilosis, however, the skin and oral mucosa has been identified as the source of infection [30,55]. This fact is of clinical importance and C. parapsilosis has been found to be increasingly implicated in BSI after placement of intravascular devices [55] and, according to the results of the investigations of Fanello, et al. the strains of Candida that come to colonize the intravascular catheters are probably the same ones that colonize skin of hands and oral mucosa [28].

More than $90 \%$ of the invasive infections due to Candida are attributed to five species: C. albicans, C. glabrata, C. parapsilosis, C. tropicalis and C. krusei. However, the list of new species of Candida isolated from clinical specimens continues to grow every year [55].

C. albicans has been the most common species of Candida isolated from BSI worldwide. However, in the past few years, there is an increasing trend of isolation of non-albicans Candida species from BSI. A number of international surveillance programs like the ARTEMIS Antifungal Surveillance program have noted a decreasing trend in the isolation of C. albicans although it still remains the most common species overall [55]. The ARTEMIS Surveillance Study which was carried out over a period of 6.5 years $(1997-2003)$ in 127 medical centres in 39 countries has shown an increase in the prevalence of Candida species like C. tropicalis (4.6\% in 1997 to $7.5 \%$ in 2003) and C. parapsilosis (4.2\% in 1997 to $7.3 \%$ in 2003) [56]. This particular surveillance study showed a 2 - to 10 -fold increase in the isolation rates of rare species like C. guillermondii, C. kefyr and C. rugosa.

C. parapsilosis is found commonly on the skin surface and has a better adherence to materials like acrylic in glucose-containing solutions and TPN solutions. It is especially known for causing BSI in infants and neonates [57]. It has been considered that the detection of Candida parapsilosis in BSI is an indication of exogenous introduction of the pathogen from the environment. However, in 2014, Batista, et al. demonstrated that the oral mucosa can also function as a route of infection in BSI in preterm infants [30]. And in 2013, a longitudinal and prospective study in which colonization patterns and dynamics were analyzed with Candida spp in infants with very low birth weight, reported that both the skin and the mucous membranes including the oropharyngeal mucosa function as pathways of infection of the bloodstream in this group of patients. The study also determined that the colonization risk at least once during the first 10 weeks was $23 \%$ for skin, $14 \%$ for oropharynx, $27 \%$ for rectum/stool, and $38 \%$ for any anatomic site sampled. The most interesting data of this study is that of the total of boys who complicated with candidemia (4/118), 75\% (3/4) of them were previously or simultaneously colonized by Candida [58]. Results similar to those reported by Leibovitz E, et al. were also announced by Singhi S, et al. in 2008. They evaluate role of Candida colonization in development of candidemia and to identify risk factors associated with Candida colonization and candidemia in children treated for severe sepsis or septic shock in a pediatric intensive care unit (PICU) for $>5$ days. And through a prospective observational study they demonstrated that the colonization by Candida species occurred in 45 (69\%) patients. Oropharyngeal (52\%) and rectal (43\%) colonization was more common than skin (34\%) colonization. The colonizing species were C. tropicalis (34.2\%), C. parapsilosis (28.8\%), C. albicans (14.4\%), and others. Use of central venous catheters was the only independent predictor of colonization on multivariate logistic regression (OR 4.1; 95\% CI 1.01-17.1). Twenty (30.2\%) patients developed candidemia; 18 (90\%) of them were colonized, 15 (75\%) with the same Candida species. Independent predictors of candidemia on multivariate stepwise logistic regression analysis were presence of colonization (OR 5.1; 95\% CI 1.0125.6, $\mathrm{p}=.048$ ) and Pediatric Risk of Mortality score (OR 1.3; 95\% CI $1.02-1.6, \mathrm{p}=.034)$ [59].

In the adult population, one study showed that oral colonization by yeast of the genus Candida is a risk factor for the onset of a nosocomial 
infection, both superficial and invasive, among hospitalized elderly subjects, establishing a colonization level $>50 \%$ ufc, as the cut-off point. ${ }^{28}$ This results of Fanello are supported with the data reported by Fong et al, in 1997 who showed that candidiasis only developed in patients with persistent asymptomatic carriage of C. albicans [60].

It is clear that, up to now, the evidence shows that colonization by Candida species, in any niche, especially in mucous membranes, is a key factor for the development of Candida infection.

Regarding the relation periodontal disease and carrying of Candida spp., Until the moment there is no global consensus, since some researchers like Hannula, et al. [61], Järvensivu, et al, [62], Urzua, et al. [34], Canabarro, et al. [63], and Rubio, et al. [29]. They report an association between Candida and periodontal disease, there may be a vicious circle, that is, the greater the periodontal commitment, the higher the prevalence of Candida, and the greater the carrying of Candida, the greater periodontal deterioration. However, there are other groups that have not identified significant differences between the prevalence and density of carrying yeasts of the genus Candida with respect to the periodontal status. The Works of Darwazeh, et al. [64], Laurenço, et al. [35], y Peters, et al. [18], are exmples of that. However, it is worth emphasizing that Laurenço's study uses a very small sample size for to see those differences. While that Peters's study have memory biased, because that classify patients according to their periodontal status based on the information they provide in a survey. In summary, these 3 papers mentioned show that both periodontally healthy and periodontally affected subjects have the same probability of complicating with a Candida infection, whether superficial or invasive, since they are in equal conditions. The variability in the results may be due to population differences, different sampling techniques, different methods for collecting the clinical data, lack of measures to control the bias, as well as non-existence in the calculation of the sample size.

Over the last ten years, some studies have found an increase in the non-albicans Candida (NAC) recovery frequency in oral mucosa: from $10 \%$ in 1996 [15] to $15.0 \%$ [ 16 ] in $2010,15.4 \%$ in 2011 [17], and $20.0 \%$ in 2017 [35] in the case of C. parapsilosis at the level of healthy subjects. Among the local and/or systemic factors associated with the higher distribution of NAC species in the oral cavity, we could mention the use of prosthetic devices [65] being elderly [66] elderly with low body mass index [67]; being an oncological patient undergoing chemotherapy or radiotherapy $[68,69]$ being a diabetic patient with poor metabolic control [70] being a man using anabolic androgenic steroids [71] or a woman using oral contraceptives [72].

Reports have indicated that C. parapsilosis and C. dubliniensis are the Candida species most frequently detected in oral niches after C. albicans among immunocompetent subjects [16,17,35,65,73,74]. Nevertheless, the epidemiology and behavior of the C. parapsilosis complex given different conditions of the oral cavity are still unknown. Our research group is the only one that has reported data on the distribution and behavior of the species that make up the C. parapsilosis complex in the oral cavity in relation to the periodontal status among immunocompetent subjects. So far we can say that among subjects with competent immunity, C. parapsilosis sensu stricto is the species most prevalent of Candida parapsilosis complex in oral niches; and the probability of recovering it increases almost 4 times, in conditions of periodontal disease [26]. This data has an epidemiological importance, since it knows the prevalence of Candida parapsilosis sensu stricto in the mouth of the healthy or immunocompetent population is $15 \%[16,17]$. which means that of every 100 subjects colonized by Candida spp in the mouth, Candida parapsilosis will be present in 15 of them, and the probability increases in conditions of gingivo-periodontal disease. However, Peters, et al. reported in 2017 a frequency of oral carrage of $80 \%$ for Candida parapsilosis sensu stricto, in the North American population; through massive sequencing technology as a method of identification and discrimination at the species level. Unfortunately, the study handled a very limited sample size ( 15 subjects per group) and this also had implicit memory bias. In any case, it is important to mention that a key datum of the Peters's study is the report of a higher oral prevalence of Candida parapsilosis with respect to Candida albicans in conditions of periodontal disease, with $80 \%$ and $60 \%$ respectively [18].

There are few studies on the oral distribution of the C. parapsilosis complex in infected HIV patients. The reports that are available come from Brazil with the work of D'Moris, et al. [75] who reported a greater recovery of Candida metapsilosis from the oral cavity in this group of subjects, followed by Candida parpasilosis sensu stricto, but without significant differences. Recently, Laurenço, et al. reported that the frequency of recovery of $C$. parapsilosis complex in the oral cavity of infected HIV subjects is only $6 \%$, with C. albicans being the predominant species in this context [35].

The virulence of Candida parapsilosis sensu stricto has been well studied both at the phenotype level and at the molecular level; and our group, until now, is the first to publish data on the virulence of oral strains of this Candida species with respect to its clinical origin. In a pilot study that our research group published in 2017, we agree with the results of Pananusorm, et al. [24] in which the biofilm forming ability in this Candida species is highly dependent on the strain, being able to distinguish strains with high, moderate, low and no biofilm forming capacity. Also finding differences regarding the clinical origin, since we identify that the strains of Candida parapsilosis sensu stricto that came from subjects with periodontal disease formed in vitro significantly more biofilm than the control group (without periodontal disease) [27]. However, it is worth mentioning that the sample size used was small, and therefore such result must be confirmed in a larger sample. But the most important data that we observed in the study was to identify a high proportion of strains that behaved as high biofilm formers, in two different nutritional conditions. From these results and the knowledge, we have about the epidemiology of candidemia, as well as periodontal disease, and the distribution of this species in the oral cavity, we wonder if the oral mucosa could be a potential source of candidemia by Candida parapsilosis sensu stricto.

According to the evidence available to date, the oral cavity under conditions of ecological equilibrium (eubiosis) will likely acquire strains of Candida parapsilosis sensu stricto (from the environment or by direct person-to-person contact) [76] with different potential for virulence; but that in a favorable environment, under ecological and immunological balance (Th1-Th2-Th17-Treg) [77] are selected in the different oral niches strains with low or no virulence capacity or symbionts strains (Figure 1). Nevertheless, if the conditions of homeostasis in the oral cavity break down, the ensuing unfavorable environment could favor the selection of more virulent strains of this Candida species or pathobionts [78] (Figure 2). A pathobiont is a microorganism which, despite expressing attributes of virulence, nevertheless continues to live in symbiosis with the host, and therefore does not cause a clinically verifiable lesion [79]. This is possible as long as the Th1-Th2-Th17-Treg balance remains intact, and fundamentally it will depend on a good Th17 response, since recent evidences show that this response is crucial for antifungal mucous defense; especially in oral mucosa $[77,78]$. When such balance breaks down, the clinical result could be oral candidiasis or invasive candidiasis. 


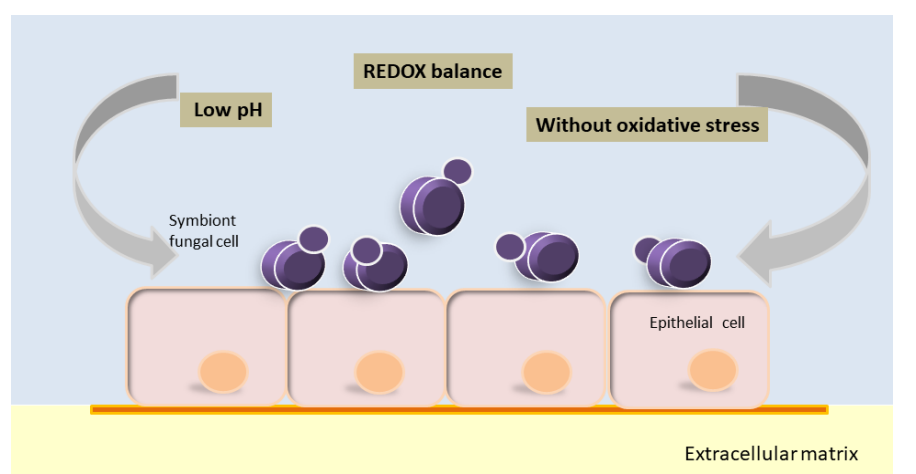

\section{Balance: Th1-Th2-Th17-Treg}

Figure 1. In conditions of ecological balance, the oral mucosa would be predominantly colonized by strains of Candida parapsilosis sensu stricto with no or low virulence capacity and in low density $[34,79]$

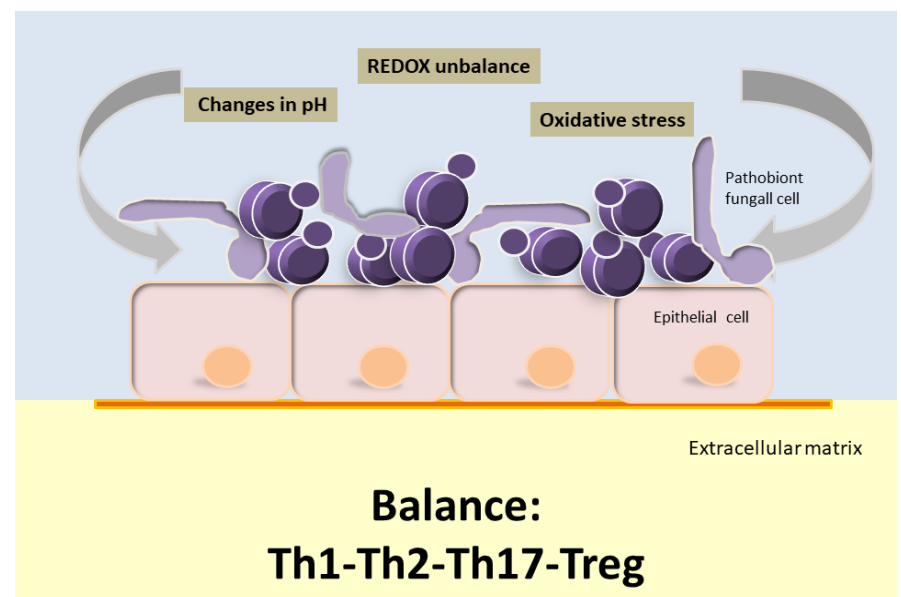

Figure 2. In conditions of oral dysbiosis, not only would the frequency and density of colonization of Candida parapsilosis sensu stricto increase, but also, the environment would favor the selection of strains with high virulence potential. However, there will be no infection until the Th1-Th2-Th17-Treg equilibrium is broken $[34,77,79]$

The advantage of this research is that for the candidemia model by Candida parapsilosis, the skin of fingers, would not be the only route of infection as the international consensus raises [1], but for everything developed in this review, we consider that the mouth would also be a possible route of infection in candidemia by this species of Candida, and even more if the oral cavity is in inflammatory conditions determined by gingivo-periodontal disease. This is particularly important from recent retrospective cohort studies, which show a significant increase in the incidence of candidemia by Candida species not Candida albicans, with Candida parapsilosis sensu stricto being the protagonist; and even more so when it is demonstrated that the morbidity and mortality due to nosocomial candidemia in preterm infants is higher than the bloodstream infections mediated by gramnegative and gram-positive bacteria [80-82].

\section{Final remarks}

We cannot draw a solid conclusion from the evidence found in the literature. A prospective study is needed to analyze the virulence of Candida parapsilosis sensu stricto according to its clinical origin at both phenotype and molecular levels. We also believe that a longitudinal study is needed to follow a cohort of subjects with and without periodontal disease over time, in order to determine how many subjects in each group develop or undergo complication with candidemia during the observational period, o al menos un studio de casos y controles. However, based on the above, it is highly likely that oral mucosa under dysbiosis is a potential source of fungemia, for three reasons:

- The dysbiosis probably influences the colonization and expression of a pathogenic phenotype in strains of Candida parapsilosis sensu stricto. To date, evidence shows that the oral cavity is more frequently colonized by strains of Candida parapsilosis sensu stricto which behave as pathobionts, but which upon encountering appropriate conditions, become pathogens, causing disease.

- Under conditions of gingival-periodontal disease, the magnitude of bacteria, yeasts and their products released into a subject's bloodstream during routine activities such as tooth brushing, dental flossing and chewing is significantly higher in time than that caused by an occasional dentistry procedure. As a result, it is likely that an oral environment in dysbiosis is a condition that promotes more frequent occurrence of candidemia events. The final outcome will depend on the fungal load present, host immunological status and microorganism virulence level.

\section{Conflicts of interest}

The authors declare no conflicts of interest.

\section{References}

1. Treviño R, Gonzáles J, Garza E, et al. (2012) Candida parapsilosis, a defiant threat (in Spanish) Candida parapsilosis, una amenaza desafiante. Medicina Universitaria 14 $157-165$

2. Tavanti A, Davidson A, Gow N, et al. (2005) Candida orthopsilosis and Candida metapsilosis spp. nov. to replace Candida parapsilosis groups II and III. J Clin Microbiol 43: 284-292.

3. Eliakim-Raz N, Babaoff R, Yahav D, et al. (2016) Epidemiology, microbiology, clinical characteristics, and outcomes of candidemia in internal medicine wards-a retrospective study. Int J Infect Dis 52: 49-54.

4. Caggiano G, Lovero G, De Giglio O, et al. (2017) Candidemia in the Neonatal Intensive Care Unit: A retrospective, observational survey and analysis of literatura data. Biomed Res Int 2017: 7901763

5. Tadec L, Talarmin J, Gastinne T (2016) Epidemiology, risk factor, species distribution, antifungal resistance and outcome of Candidemia at a single French hospital: a 7-year study. Mycoses 59: 296-303.

6. Tan T, Hsu L, Alejandria M (2016) Antifungal susceptibility of invasive Candida bloodstream isolates from the Asia-Pacific region. Med Mycol 54: 471-477.

7. Nucci M, Queiroz-Telles F, Alvarado-Matute T, Tiraboschi IN, Cortes J, et al. (2013) Epidemiology of candidemia in Latin America: a laboratory-based survey. PLoS One 8: e59373. [Crossref]

8. Kullberg BJ, Arendrup MC (2016) Invasive Candidiasis. N Engl J Med 374: 794-795 [Crossref]

9. Falcone M, Concia E, Iori I, et al. (2014) Identification and management of invasive mycoses in internal medicine: a road-map for phy-sicians. Intern Emerg Med 9: 50111. 3 .

10. Zaoutis TE, Argon J, Chu J, Berlin JA, Walsh TJ, et al. (2005) The epidemiology and attributable outcomes of candidemia in adults and children hospitalized in the United States: a propensity analysis. Clin Infect Dis 41: 1232-1239. [Crossref]

11. Weinberger M, Sweet S, Leibovici L, Pitlik SD, Samra Z (2003) Correlation between candiduria and departmental antibiotic use. J Hosp Infect 53: 183-186. [Crossref] 
12. Ben-Ami R, Olshtain-Pops K, Krieger M, et al. Israeli Candidemia Study Group. Antibiotic exposure as a risk factor for fluconazole-resistant Candida bloodstream infection. Antimicrob Agents Chemother 56: 2518-2523.

13. Bassetti M, Merelli M, Righi E, et al. (2013) Epidemiology, species distribution, antifungal susceptibility, and outcome of candidemia across five sites in Italy and Spain. J Clin Microbiol 51: 4167-4172.

14. Pfaller MA, Castanheira M, Messer SA, Moet GJ, Jones RN (2010) Variation in Candida spp. distribution and antifungal resistance rates among bloodstream infection isolates by patient age: report from the SENTRY Antimicrobial Surveillance Program (2008-2009). Diagn Microbiol Infect Dis 68: 278-283. [Crossref]

15. Kleinegger CL, Lockhart SR, Vargas K, Soll DR (1996) Frequency, intensity, species, and strains of oral Candida vary as a function of host age. J Clin Microbiol 34: 22462254. [Crossref]

16. Ghannoum MA, Jurevic RJ, Mukherjee PK, Cui F, Sikaroodi M, et al. (2010) Characterization of the oral fungal microbiome (mycobiome) in healthy individuals. PLoS Pathog 6: e1000713. [Crossref]

17. Yang Y, Leaw S, Wang A, et al. (2011) Characterization of yeasts colonizing in healthy individuals. Medical Mycology 49: 103-106.

18. Peters BA, Wu J, Hayes RB, et al. (2017) The oral fungal mycobiome: characteristics and relation to periodontitis in a pilot study. BMC Microbiol 17: 157. [Crossref]

19. Trofa D, Gácser A, Nosanchuk JD (2008) Candida parapsilosis, an emerging fungal pathogen. Clin Microbiol Rev 21: 606-625. [Crossref]

20. Ge Y, Boekhout T, Zhan P, et al. Characterization of the Candida parapsilosis complex in East China: species distribution differs among cities. Medical Mycology 50: 56-66.

21. Miranda I, Eraso E, Hernández J, et al. (2011) Prevalence and antifungal susceptibility patterns of new cryptic species inside the species complexes Candida parapsilosis and Candida glabrata among blood isolates from a Spanish tertiary hospital. J Antimicrob Chemother 66: 2315-2322.

22. Silva A, Miranda I, Lisboa C, et al. (2009) Prevalence, Distribution, and Antifunga Susceptibility Profiles of Candida parapsilosis, C. orthopsilosis, and C. metapsilosis in a Tertiary Care Hospital. Journal of Clinical Microbiology 47: 2392-2397.

23. Tosun I, Akyuz Z, Guler N, et al. (2012) Distribution, virulence attributes and antifungal susceptibility patterns of Candida parapsilosis complex strains isolated from clinical samples. Medical Mycology 51: 483-492.

24. Pannanusorn S, Ramírez B, Lünsdorfet H, et al. Characterization of Biofilm Formation and the Role of BCR1 in Clinical Isolates of Candida parapsilosis. Eukaryotic Cell 13: 438-451.

25. Constante C, Monteiro A, Alves S, et al. Different risk factors for candidemia occur for Candida species belonging to the C. parapsilosis complex. Medical Mycology 52: 403-406.

26. Rodríguez L, Jewtuchowicz V (2016) Molecular characterization of Candida parapsilosis species complex in niches of the oral cavity in a cohort of patients from Argentina with different oral and dental clinical manifestations. J Dent Sci Ther 1: $18-25$.

27. Rodríguez L, Rosa A, Rodríguez J, et al. (2017) The Oral Cavity: A Reservoir that Favors Colonization and Selection of Candida parapsilosis sensu stricto Strains with High Pathogen Potential Under Conditions of Gingival-periodontal Disease. J Dent Sci Ther 2 .

28. Fanello S, Bouchara J, Sauteron M. et al. (2006) Predictive value of oral colonization by Candida yeasts for the onset of a nosocomial infection in elderly hospitalized patients. J Med Microbiol 55: 223-228.

29. Rubio N, Puia S, Toranzo S, et al. (2015) Fungal invasion of connective tissue in patienst with gingival-periodontal disease. Revista Iberoamericana de Micología 32: 20-24.

30. Batista GC, Krebs VL, Ruiz LS, Auler ME, Hahn RC, et al. (2014) Oral colonization: a possible source for candidemia in low-weight neonates. $J$ Mycol Med 24: 81-86. [Crossref]

31. El-Masry FA, Neal TJ, Subhedar NV (2002) Risk factors for invasive fungal infection in neonates. Acta Paediatr 91: 198-202. [Crossref]

32. Carey A, Saiman L, Polin R (2008) Hospital-acquired infections in the NICU: epidemiology for the new millennium. Clin Perinatol 35: 223-249.

33. Falagas M, Rousssos N, Vardakas K (2010) Relative frecuency of albicans and the various non-albicans Candida spp among candidemia isolates from inpatients in various parts of the world: a systemactic review. Int J Infect Dis 14: 954-966.
34. Urzua B, Hermosilla G, Gamonal J, et al. (2008) Yeast diversity in the oral microbiota of subjects with periodontitis: Candida albicans and Candida dubliniensis colonize the periodontal pockets. Medical Mycology 46: 783-793.

35. Louren A, Rodrigues A, Nakao C, et al. (2017) Oral Candida spp carriage and periodontal disease in HIV-infected patients in Ribeirão Preto, Brazil. Rev Inst Med trop S Paulo 59: e29.

36. Kinane DF, Stathopoulou PG, Papapanou PN (2017) Periodontal diseases. Nat Rev Dis Primers 3: 17038. [Crossref]

37. Michaud DS, Fu Z, Shi J, Chung M (2017) Periodontal Disease, Tooth Loss, and Cancer Risk. Epidemiol Rev 39: 49-58. [Crossref]

38. Ezzati M, Riboli E (2012) Can noncommunicable diseases be prevented? Lessons from studies of populations and individuals. Science 337: 1482-1487. [Crossref]

39. FDI World Dental Federation (2013a) FDI policy statement on non-communicable diseases. International Dental Journal 63: 285-286.

40. Jin L (2013) The global call for oral health and general health. Int Dent J 63: 281-282. [Crossref]

41. Sheiham A, Watt RG (2000) The common risk factor approach: a rational basis for promoting oral health. Community Dent Oral Epidemiol 28: 399-406. [Crossref]

42. United Nations General Assembly. Political Declaration of the High-level Meeting of the General Assembly on the Prevention and Control of Non-communicable Diseases. 2011. Available at: http://www.un.org/ga/search/view_doc.asp?symbol=A/66/L.1

43. Darveau, R (2010) Periodontitis: A polymicrobial disruption of host homeostasis. Nature Reviews Microbiology 8: 481-490.

44. Hajishengallis G, Liang S, Payne M, et al. (2011) Low-abundance biofilm species orchestrates inflammatory periodontal disease through the commensal microbiota and complement. Cell Host \& Microbe 10: 497-506.

45. Kornman KS (2008) Mapping the pathogenesis of periodontitis: a new look. $J$ Periodontol 79: 1560-1568. [Crossref]

46. Sanz M, Beighton D, Curtis M, et al (2017) Role of microbial biofilms in the maintenance of oral health and in the development of dental caries and periodontal diseases. Consensus report of group 1 of the Joint EFP/ORCA workshop on the boundaries between caries and periodontal disease. Journal of Clinical Periodontology 44: S5-S11.

47. Buset S, Walter C, Friedmann A, et al. (2016) Are periodontal diseases really silent? A systematic review of their effect on quality of life. Journal of Clinical Periodontology 43: 333-344.

48. Sheiham A, Watt R (2000) The common risk factor approach: A rational basis for promoting oral health. Community Dentistry and Oral Epidemiology 28: 399-406.

49. Kassebaum N, Bernabé E, Dahiya M, et al. (2014) Global burden of severe tooth loss: A systematic review and meta-analysis. Journal of Dental Research 93: 20S-28S.

50. Tonetti M, Bottenberg P, Conrads G, et al. (2017) Dental caries and periodontal diseases in the ageing population: call to action to protect and enhance oral health and wellbeing as an essential component of healthy ageing - Consensus report of group 4 of the joint EFP/ORCA workshop on the boundaries between caries and periodontal diseases. Journal of Clinical Periodontology 44: S135-S144.

51. Wilson W, Taubert K, Gewitz M, et al. (2007) Prevention of Infective Endocardit Guidelines From the American Heart Association A Guideline From the American Heart Association Rheumatic Fever, Endocarditis, and Kawasaki Disease Committee, Council on Cardiovascular Disease in the Young, and the Council on Clinical Cardiology, Council on Cardiovascular Surgery and Anesthesia, and the Quality of Care and Outcomes Research Interdisciplinary Working Group. Journal of the American Heart Association.

52. Debelian G, Olsen I, Tronstad L (1998) Anaerobic Bacteremia and Fungemia in Patient Undergoing Endodontic Therapy: An Overview. Annals of Periodontology 3: 281-287.

53. Wisplinhoff H, Bischoff T, Tallent S, et al. (2004) Nosocomial bloodstream infections in US hospitals: Analysis of 24,179 cases from a prospective nationwide surveillance study. Clin Infect Dis 39: 309-317.

54. Giri S, Kindo A (2012) A review of Candida species causing blood stream infection. Indian Journal of Medical Microbiology 30: 270-278.

55. Pfaller MA, Diekema DJ (2007) Epidemiology of invasive candidiasis: A persistent public health problem. Clin Microbiol Rev 20: 133-163. [Crossref]

56. Pfaller MA, Diekema DJ, Rinaldi MG, Barnes R, Hu B, Veselov AV (2005) Results from the ARTEMIS DISK Global antifungal surveillance study: A 6.5-year analysis of susceptibilities of Candida and other yeast species to fluconazole and voriconazole by standardized disk diffusion testing. J Clin Microbiol 43: 5848-5859. 
57. Levy I, Rubin LG, Vasishtha S, Tucci V, Sood SK (1998) Emergence of Candida parapsilosis as the predominant species causing candidemia in children. Clin Infect Dis 26: 1086-1088. [Crossref]

58. Leibovitz E, Livshiz-Riven I, Borer A, et al. (2013) A prospective study of the patterns and dynamics of colonization with Candida spp. in very low birth weight neonates. Scand J Infect Dis 45: 842-848.

59. Singhi S, Rao DS, Chakrabarti A (2008) Candida colonization and candidemia in a pediatric intensive care unit. Pediatr Crit Care Med 9: 91-95. [Crossref]

60. Fong IW, Laurel M, Burford-Mason A (1997) Asymptomatic oral carriage of Candida albicans in patients with HIV infection. Clin Invest Med 20: 85-93. [Crossref]

61. Hannula J, Dogan B, Slots J (2001) Subgingival strains of Candida albicans in relation to geographical origin and occurrence of periodontal pathogenic bacteria. Oral Microbiol Immunol 16: 113-118.

62. Järvensivu A, Hietanen J, Rautemaa R (2004) Candida yeasts in chronic periodontitis tissues and subgingival microbial biofilms in vivo. Oral Dis 10: 106-112.

63. Canabarro A, Valle C, Farias MR (2013) Association of subgingival colonization of Candida albicans and other yeasts with severity of chronic periodontitis. $J$ Periodontal Res 48: 428-432.

64. Darwazeh AM, Hammad MM, Al-Jamaei AA (2010) The relationship between oral hygiene and oral colonization with Candida species in healthy adult subjects*. Int $J$ Dent Hyg 8: 128-133. [Crossref]

65. Jewtuchowicz V, Brusca M, Mujica M, et al. (2007) Subgingival distribution of yeast and their antifungal susceptibility in immunocompetent subjects with and without dental devices. Acta Odontol Latinoam 20: 17-22

66. Benito B, Aranda S, López F, de la Rosa E, Rosas R, Sánchez L (2016) Oral Candida isolates and fluconazole susceptibility patterns in older Mexican women. Arch. Gerontol Geriatr 9: 204-210.

67. Zakaria MN, Furuta M, Takeshita T, et al. (2017) Oral mycobiome in communitydwelling elderly and its relation to oral and general health conditions. Oral Dis 23: 973-982. [Crossref]

68. Jain M, Shah R, Chandolia B, et al. (2016) The oral carriage of Candida in oral cancer patients of indian origin undergoing radiotherapy and/or chemotherapy. J Clin Diagn Res 10: $17-20$

69. Bulacio L, Paz M, Ramadan S, et al. (2012) Oral infections caused by yeasts in patients with head and neck cancer undergoing radiotherapy. Identification of the yeasts and evaluation of their antifungal susceptibility. J Mycol Med 22: 348-353.
70. Melton J, Redding S, Kirkpatrick W, et al. (2010) Recovery of Candida dubliniensis and other Candida species from the oral cavity of subjects with periodontitis who had well-controlled and poorly controlled type 2 diabetes: a pilot study. Special Care in Dentistry 30: 230-234.

71. Brusca MI, Verdugo F, Amighini C, Albaina O, Moragues MD (2014) Anabolic steroid affect human periodontal health and microbiota. Clin Oral Investig 18: 1579-1586. [Crossref]

72. Brusca M, Rosa A, Olatz A, Moragues M, Verdugo F, Pontón J (2010) The Impact of Oral Contraceptives on Women's Periodontal Health and the Subgingival Occurrence of Aggressive Periodontopathogens and Candida Species. Journal of Periodontology 81: 1010-1018.

73. Bertone A, Rosa A, Nastri N, et al. (2016) Genetic-related of peri-implants and buccal Candida albicans isolates determined by RAPD-PCR. Acta Odontol. Latinoam 29: 197 205 .

74. Mushi M, Mtemisika C, Bader O, et al. (2016) High oral carriage of non-albicans Candida spp. among HIV infected individuals. International Journal of Infectious Disease 49: 185-188.

75. Moris S, Melhem S, Martins M, et al. (2012) Prevalence and antifungal susceptibility of Candida parapsilosis complex isolates collected from oral cavities of HIV-infected individuals. Journal of Medical Microbiology 61: 1758-1765.

76. Fanello S, Bouchara J, Jousset N. et al. (2001) Nosocomial Candida albicans acquisition in a geriatric unit: epidemiology and evidence for person-to-person transmission. $J$ Hosp Infect 47: 46-52.

77. Richardson J, Moyes D (2015) Adaptive immune responses to Candida albican infection. Virulence 6: 327-337.

78. Gow N, van de Veerdonk F, Brown A, et al. (2012) Candida albicans morphogenesis and host defence: discriminating invasion from colonization. Nature review microbiology 10: $112-122$.

79. Hornef M (2015) Pathogens, commensal symbionts, and pathobionts: Discovery and functional effects on the host. ILAR J 56: 159-162. [Crossref]

80. Ghezzi M, Brunetti G, Visconti V, et al. (2017) Candidaemia in a tertiary care academic hospital in Italy. The impact of C. parapsilosis complex on the species distribution and antifungal susceptibility. J Med Microbiol 66: 990-998.

81. Caggiano G, Lovero G, De Giglio O, et al. (2017) Candidemia in the Neonatal Intensive Care Unit: A Retrospective, Observational Survey and Analysis of Literature Data. Biomed Res Int 2017: 7901763.

82. Tiraboschi I, Pozzi N, Farías L, et al. (2017) Epidemiology, species, antifungal resistance and outcome of candidemia in a university hospital in Buenos Aires, Argentina for 16 years. Rev Chilena Infectol $34: 431-440$.

Copyright: @2019 Rodríguez ML. This is an open-access article distributed under the terms of the Creative Commons Attribution License, which permits unrestricted use, distribution, and reproduction in any medium, provided the original author and source are credited. 